\title{
Sole regional anaesthetic technique for a through knee amputation in a patient with spina bifida.
}

\author{
Khafaga M* \\ Countess of Chester Hospital NHS Foundation Trust, Chester, UK
}

\begin{abstract}
Introduction: A 44-year-old lady with a history of spina bifida, chronic renal failure and grade 4 intubation needing an awake fiber-optic presented for a through knee amputation. A decision was taken to follow a sole regional anaesthetic and postoperative analgesia plan.

Methods: Femoral block: Lateral approach in plane technique was adopted and $10 \mathrm{ml}$ of chiroccaine $0.5 \%$ added to $10 \mathrm{ml}$ of lignocaine $1 \%$ were injected to completely surround the femoral nerve. Sciatic sub gluteal block: Lateral approach in plane technique was adopted and $10 \mathrm{ml}$ of chiroccaine $0.5 \%$ added to $10 \mathrm{ml}$ of lignocaine $1 \%$ were injected to completely surround the sciatic nerve. Adductor canal block: Lateral approach in plane technique was adopted and $10 \mathrm{ml}$ of chiroccaine $0.5 \%$ added to $10 \mathrm{ml}$ of lignocaine $1 \%$ were injected to completely surround the femoral artery in the adductor canal.

Discussion: In view of the multiple comorbidities including the spina bifida, the renal disease and the history of difficult airway management, we decided to perform a sole regional anaesthetic technique for intraoperative and postoperative analgesia. The patient already had a patchy sensation over the lower limbs due to her neurological condition, which was another factor in favour of the choice of the anaesthetic plan. The operation was safely performed with the patient being very comfortable without needing any further sedation.

Conclusion: Sole regional anaesthetic techniques could be used successfully for lower limb surgeries in patients suffering from Spina Bifida.
\end{abstract}

Keywords: Regional anaesthetic, Spina biida, Knee amputation, Fiberoptic.

Accepted on April 13, 2019

\section{Introduction}

A 44-year-old lady with a history of spina bifida, chronic renal failure and grade IV intubation needing an awake fiberoptic, presented for a through knee amputation. This is a challenging case and a procedure which needed a specific anaesthetic plan. A decision was taken to follow a sole regional anaesthetic and postoperative analgesic plan.

Neural tube defects (NTDs) are congenital malformations of the brain and spinal cord in neurulation that occur between 21 and 28 days after conception. The most common subtypes of cases include spina bifida, anencephaly and encephalocele. The disease is one of the most prevalent and most severe of birth defects with a high mortality rate. As reported, the average worldwide prevalence is 1 per 1000 living birth, in whites it is approximately the same. Spina bifida is a type of neural tube defect. The neural tube is the structure that eventually develops into the baby's brain and spinal cord. The neural tube starts to form in early pregnancy and closes about 4 weeks after conception. In spina bifida, part of the neural tube doesn't develop or close properly, leading to defects in the spinal cord and bones of the spine (vertebrae). It's not known what causes spina bifida, but a lack of folic acid before and in the early stages of pregnancy is a significant risk factor [1].
Symptoms of spina bifida are: Weakness or total paralysis of the legs, bowel incontinence and urinary incontinence, loss of skin sensation in the legs and around the bottom-the patient is unable to feel hot or cold, which can lead to accidental injury [2].

Some of these patients have a ventriculo-peritoneal shunt for hydrocephalus. All patients with Ventriculoperitoneal (VP) shunt require neurosurgical review and a Computed tomography (CT) scan before their general anaesthetic if their shunt status is not known. A poor history and lack of neurosurgical follow-up must be an indication for neurosurgical referral. This is recommended even for asymptomatic patients coming in for short procedures under General anaesthesia (GA). A neurosurgeon would be able to assess the shunt and if required insert a new one. A poorly assessed shunt could be a death trap and a hurried GA is not advised $[3,4]$.

These patients may have no neurological symptoms or may have minor motor and sensory deficits of the lower limbs, bladder and bowel. However, tethering of the spinal cord is present in 35 $87 \%$ of patients with spinal dysraphism and this has implications for the ability to carry out regional anaesthetic techniques [5].

Spina bifida occulta (a subtype of spina bifida) represents a challenge for epidural anaesthesia technique, poorly reported in literature. Nevertheless, known benefits of epidural anaesthesia for labour make the adaptation of this technique 
in this challenging situation preferable to general anaesthesia. As shown, epidural anaesthesia may be still feasible in patients with spina bifida occulta [6].

The literature is really deficient in information about peripheral nerves blocks in spina bifida patients, hence the importance of this case report where a peripheral nerve block was successfully given to a patient with spina bifida for a lower limb surgery.

\section{Discussion}

A 44-year-old lady scheduled for a left through knee amputation, to be done by the vascular team. The interesting thing about the patient is her rare and unique background, as well as her comorbidities and the adopted anaesthetic plan which seemed to be perfectly suitable to her condition. She weighs 48 $\mathrm{kg}$ and measures 1.5 meters. As a medical history, she is known to have a spina bifida and hydrocephaly since birth with loss of motor function in both lower limbs as well as patchy sensory loss over both lower limbs, mild asthma controlled by medications (Figure 1). She has chronic kidney disease stage 3 and she previously had a hypo-calcaemic tetany. She has got factor 5 Leiden and deep venous thrombosis back in 2006 in her right leg. She has got chronic anaemia treated with iron infusions. Concerning her surgical history she had a right nephrectomy in 2003 for a staghorn calculus. She has a cystostomy with a stoma in the abdomen and she self-catheterizes 5 times per day. As regard to her medications, she is on vit D, alphacalcidol, ferrous sulphate, loperamide, lansoprazole, sodium bicarbonate, funoximide, qvar, salbutamol, and irbesartan.

\section{Airway assessment}

Mallampati score 3, mouth opening 3 fingers, thyromental distance $<6.5 \mathrm{~cm}$, normal cranio-cervical mobility, small receding mandible. She is known to be grade IV intubation and needed an awake fiber-optic intubation. She is not a smoker and does not drink any alcohol. She is wheelchair bound.

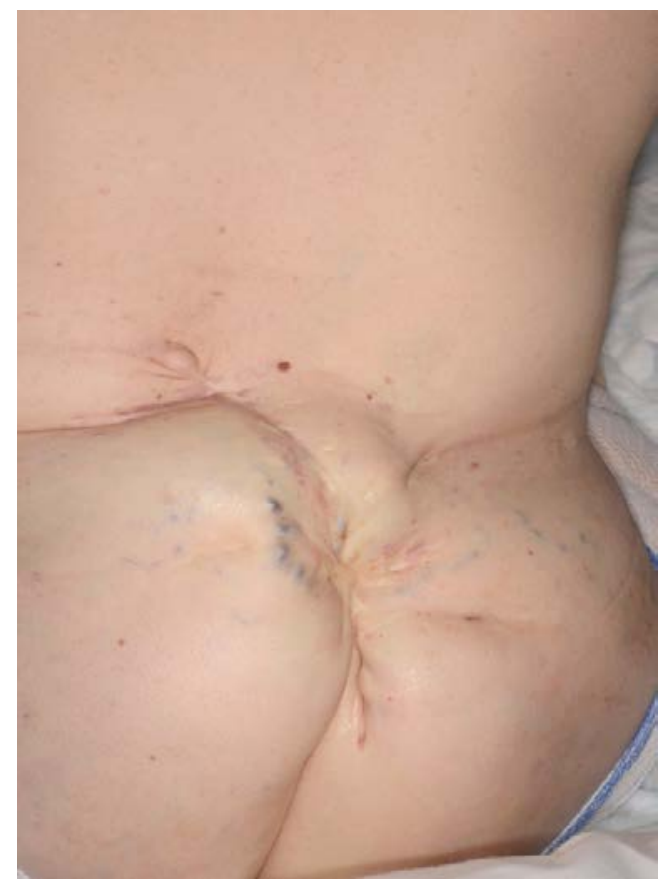

Figure 1. The patient's back with the spina bifida deformity.

\section{Anaesthetic plan}

Preoperative: Review of routine investigations, discussion of Venous Thromboembolism (VTE) prophylaxis with the haematology specialty registrar (SPR).

Intraoperative: Femoral and a sciatic sub-gluteal blocks and intraoperative IV Calcium.

Postoperative: VTE prophylaxis, pain control.

\section{Perioperative management}

The patient came into the anaesthetic room on her bed. Routine monitoring was attached (electrocardiogram, noninvasive blood pressure, pulse oximetry). Oxygen mask was placed on the patient's face. Intravenous access was secured through the insertion of an $18 \mathrm{G}$ intravenous catheter on the right hand.

Femoral nerve block: The patient was lying flat, left groin cleaned with $0.5 \%$ chlorhexidine, sterile gloves were used, sterile gel was used, a covered linear ultrasound probe was used to scan the groin and identify the femoral nerve. Three milliliters of lignocaine $2 \%$ were injected subcutaneously and then a 50 mm stimuplex needle was used to inject the local anaesthetics. Lateral approach in plane technique was adopted and $10 \mathrm{ml}$ of chiroccaine $0.5 \%$ added to $10 \mathrm{ml}$ of lignocaine $1 \%$ were injected to completely surround the femoral nerve.

Sciatic sub gluteal block: The patient was lying on her right side, the left sub gluteal region was cleaned with $0.5 \%$ chlorhexidine, sterile gloves were used, sterile gel was used, a covered curved ultrasound probe was used to scan the region and identify the Sciatic nerve. $3 \mathrm{ml}$ of lignocaine $2 \%$ were injected subcutaneously and then a $100 \mathrm{~mm}$ stimuplex needle was used to inject the local anaesthetics. Lateral approach in plane technique was adopted and $10 \mathrm{ml}$ of chiroccaine $0.5 \%$ added to $10 \mathrm{ml}$ of lignocaine $1 \%$ were injected to completely surround the sciatic nerve. Sciatic nerve block provides postoperative pain relief after below knee amputation, knee replacement, foot, and ankle surgery [7].

Adductor canal block: The patient was lying flat, the left medial aspect of the thigh was cleaned with $0.5 \%$ chlorhexidine, sterile gloves were used, sterile gel used, a covered linear ultrasound probe was used to scan the mid-thigh region and identify adductor canal. $3 \mathrm{ml}$ of lignocaine $2 \%$ were injected subcutaneously and then a $50 \mathrm{~mm}$ stimuplex needle was used to inject the local anaesthetics. Lateral approach in plane technique was adopted and $10 \mathrm{ml}$ of chiroccaine $0.5 \%$ added to $10 \mathrm{ml}$ of lignocaine $1 \%$ were injected to completely surround the femoral artery in the adductor canal.

Sensory testing was done with the use of ethyl alcohol spray and sensation was reasonably blocked.

The surgeons started skin disinfection and draping. Skin sensation was tested again before incision. Incision done and through knee amputation performed with the patient really comfortable, no sedation needed to be given. Blood loss was approximately $200 \mathrm{ml}$ and $500 \mathrm{ml}$ of hartman's solution were 
given. $10 \mathrm{ml}$ of $10 \%$ calcium gluconate were given slowly intravenously as a prophylaxis. The pain control was perfect and the patient was conscious all through the procedure.

Postoperative management: Postoperative analgesia was prescribed for the patient as well as venous thromboembolism prophylaxis.

\section{Conclusion}

Sole regional anaesthetic techniques, like femoral and sciatic nerve block, could be used successfully for lower limb surgeries in patients suffering from spina bifida.

\section{References}

1. Zhang T, Lou J, Zhong R, et al. Genetic variants in the folate pathway and the risk of neural tube defects: a meta-analysis of the published literature. PLoS One. 2013;8(4):e59570.
2. https://www.nhs.uk/conditions/Spina-bifida

3. Martín MC, Pagès L, Raduá, V, et al. The spina bifida occulta challenge for anaesthesia during labor. Eur J Anaesthesiol. 2012;29(1):165.

4. Griffiths S, Durbridge JA. Anaesthetic implications of neurological disease in pregnancy. Continuing Educ Anaesth Crit Care Pain. 2011;11(5):157-61.

5. Ali L, Stocks GM. Spina bifida, tethered cord and regional anaesthesia. Anaesthesia. 2005;60(11):1149-50.

6. Beard L, Downs A. An unexpected lumbar lesion. J Obstet Anaesth Crit Care. 2016;6(1):104-6.

7. Munirama S, McLeod G. Ultrasound-guided femoral and sciatic nerve blocks. Continuing Educ Anaesth Crit Care Pain. 2013;13(4):136-40.

\section{*Correspondence to:}

Khafaga $\mathrm{M}$

Countess of Chester Hospital NHS Foundation Trust Chester

UK

Tel: + 447490388824

E-mail: dr.m.khafaga@gmail.com 